COLON CANCER

\title{
Efficacy of high magnification chromoscopic colonoscopy for the diagnosis of neoplasia in flat and depressed lesions of the colorectum: a prospective analysis
}

\author{
D P Hurlstone, S S Cross, I Adam, A J Shorthouse, S Brown, D S Sanders, A J Lobo
}

Gut 2004;53:284-290. doi: 10.1136/gut.2003.027623

See end of article for authors' affiliations

Correspondence to: Dr D P Hurlstone, 17 Alexandra Gardens, Lyndhurst Rd, Nether Edge, Sheffield S119DQ, UK; p.hurlstone@ shef.ac.uk

Accepted for publication 17 September 2003

\begin{abstract}
Background: High magnification chromoscopic colonoscopy (HMCC) permits the in vivo examination of the colorectal pit pattern, which has a high correlation with stereomicroscopic appearances of resected specimens. This new technology may provide an "optical biopsy" which can be used to aid diagnostic precision and guide therapeutic strategies. Conflicting data exist concerning the accuracy of this technique when discriminating neoplastic from non-neoplastic lesions, particularly when flat and depressed.

Aim: To prospectively examine the efficacy of HMCC for the diagnosis of neoplasia in flat and depressed colorectal lesions using standardised morphological, pit pattern, and histopathological criteria. Clinical recommendations for the use of HMCC are made.

Methods: Total colonoscopy was performed on 1850 patients by a single endoscopist from January 2001 to July 2003 using the C240Z magnifying colonoscope. Identified lesions were classed according to the Japanese Research Society guidelines, and pit pattern according to Kudos modified criteria. Pit pattern appearances were then compared with histopathology.

Results: A total of 1008 flat lesions were identified. The sensitivity and specificity of HMCC in distinguishing non-neoplastic from neoplastic lesions were $98 \%$ and $92 \%$, respectively. However, when using HMCC to differentiate neoplastic/non-invasive from neoplastic/invasive lesions, sensitivity was poor $(50 \%)$ with a specificity of $98 \%$. Diagnostic accuracy was not influenced by size or morphological classification of lesions.

Conclusion: HMCC has a high overall accuracy at discriminating neoplastic from non-neoplastic lesions but is not 100\% accurate. HMCC is a useful diagnostic tool in vivo but presently is not a replacement for histology. Requirements for further education and training in these techniques need to be addressed.
\end{abstract}

$\mathrm{T}$ he secondary prevention of colorectal cancer (CRC) assumes that early detection and resection of precursor lesions will disrupt the adenoma-carcinoma sequence and halt progression to invasive neoplastic disease. ${ }^{1}$ The adenoma-carcinoma sequence described by Morson has until now formed the rationale for endoscopic therapies directed at reducing the incidence of CRC. ${ }^{2}$ The fact that snare polypectomy of exophytic lesions fails to prevent progression to carcinoma in up to $24 \%$ of lesions ${ }^{3}$ has prompted many authors to re-evaluate the prevalence and clinicopathological significance of flat and depressed colorectal lesions in Western cohorts. Such lesions, although well described by the Japanese, ${ }^{45}$ have only recently been reported in the West. ${ }^{6-9}$ Controversy has existed regarding their prevalence, anatomical localisation, and histopathological characteristics. We recently reported in a large prospective study in the UK that flat lesions accounted for $38 \%$ of all lesions, where $82 \%$ of flat lesions with high grade dysplasia (HGD) and $90 \%$ of all flat/depressed carcinomas were located in the right colon. ${ }^{6}$ These data supported similar trends reported in other series ${ }^{7-11}$ and stresses the importance of detection and definitive endoscopic therapy, particularly given the imminent introduction of a nationwide CRC screening programme in the UK.

High magnification chromoscopic colonoscopy (HMCC) permits the in vivo examination of the colorectal surface crypt or pit pattern, which has a high correlation with stereomicroscopic appearances of resected specimens. ${ }^{12}{ }^{13}$ The premise of this technology is to provide an in vivo optical biopsy that can be used at the time of colonoscopy to enhance diagnostic precision and guide subsequent therapeutic strategies.

Regarding exophytic or polypoid lesions, HMCC is not required, as there is an established and validated correlation between size and neoplastic risk, which is the major consideration when choosing endoscopic snare polypectomy or surgical resection. ${ }^{14-16}$ However, flat and depressed colorectal lesions do not conform to this basic rationale, where therapeutic decisions are highly dependent on the detailed morphological appearance, including the pit pattern. ${ }^{417} 18$ Indeed, some authors propose such lesions may favour a de novo pathogenic pathway where early submucosal invasion and risk of associated lymph node disease can occur. ${ }^{17} 1920$

Conflicting data concerning the sensitivity, specificity, and overall accuracy of HMCC have additionally become apparent in the setting of routine clinical practice. ${ }^{13} 2122$ Variability in these data is multifactorial, being in part related to operator experience, ${ }^{2122}$ chromoscopic technique, ${ }^{23}$ and East-West ambiguity in morphological and histopathological classification. $^{24}$ We therefore prospectively examined the efficacy of HMCC for the diagnosis of neoplasia in flat and depressed colorectal lesions using standardised morphological, pit pattern, and histopathological criteria.

Abbreviations: $\mathrm{CRC}$, colorectal cancer; $\mathrm{HMCC}$, high magnification chromoscopic colonoscopy; HGD, high grade dysplasia; LGD, low grade dysplasia; NSS, normal saline solution; IC, indigo carmine; CV, crystal violet; EMR, endoscopic mucosal resection; USMP, ultrasound mini probe 


\section{METHODS}

Total colonoscopy was performed prospectively on 1850 patients using the Olympus $\mathrm{C} 240 \mathrm{Z}$ magnifying colonoscope, from January 2001 to July 2003, by a single endoscopist. Full ethics approval for the study was obtained from the South Sheffield Research Ethics Committee. Signed informed consent was obtained from all participating patients. The caecum was identified by the appendix orifice, tri-radiate caecal fold, ileocaecal valve, and terminal ileal or neoterminal-ileal intubation with small bowel biopsy in patients with a previous right hemicolectomy. Bowel preparation consisted of 2-4 litres of hypertonic polyethylene glycol solution (Kleanprep) 24 hours prior to the procedure.

\section{Inclusion/exclusion criteria}

Patients were selected for inclusion to the study if they were considered "high risk" candidates for underlying colorectal polyps or neoplasia. Exclusion criteria were familial adenomatous polyposis, known advanced CRC, acute severe inflammatory bowel disease, none correctable coagulopathy, poor bowel preparation, and inability to give informed consent.

\section{Endoscopic mucosal evaluation}

All lesions were initially identified using conventional video colonoscopy by direct visualisation or recognition of the following subtle mucosal signs ${ }^{25}$ :

- focal pallor or erythema (fig $1 \mathrm{~A}-\mathrm{C}$ );

- haemorrhagic spots;

- fold convergence;

- disruption of the mucosal vascular net pattern (fig 2A-C);

- unevenness or discrete mucosal deformity;

- air induced deformation.

Following identification, all suspected lesions were washed with a $10-20 \mathrm{ml}$ bolus of normal saline solution (NSS) introduced down the side port of the colonoscope. The lesion contour was then delineated using $0.5 \%$ indigo carmine (IC) solution $(2-5 \mathrm{ml})$ flushed down the side port followed by a $20 \mathrm{ml}$ air "push".

\section{Morphological classification of lesions}

The detailed non-magnified chromoscopic appearances of all lesions was documented using the established macroscopic classification of the Japanese Research Society of Cancer of the Colon and Rectum. ${ }^{26}{ }^{27}$ Flat lesions were defined as those with no distinct stalk or pedicle with a flat or rounded surface where the height of the lesion was less than half the diameter ${ }^{28}$ The size of each lesion was estimated using a fully opened standard biopsy forcep (4 mm) (Bard Inc., USA) with height estimated by placing the closed forcep $(2 \mathrm{~mm})$ adjacent to the lesion.

\section{High magnification examination and pit pattern classification}

Following morphological classification using IC chromoscopy, all lesions underwent magnification examination $(100 \times$ normal) by depression of the thumb activated lever adjacent to the up/down axis control of the C240Z colonoscope. The surface pit pattern was then classified according to the modified Kudo criteria (type I-V) (table 1). ${ }^{12}$ In addition, when a discrete type I-IV pattern could not be identified or there was evidence of central depression (as evidenced by mucosal pooling of IC) (fig $3 \mathrm{~A}-\mathrm{C}$ ), the lesion was further assessed using crystal violet (CV) chromoscopy.

CV is an absorptive stain used to accurately differentiate the presence of the invasive type $\mathrm{V}$ pit pattern. ${ }^{25}$ This is clinically important as this pattern, in association with central depression, can signify the presence of cancer with deep submucosal invasion. ${ }^{42}$ Prior to CV chromoscopy, the lesion was therefore subsequently washed again with NSS and any surface mucous removed by the local application of 2-4 ml $\mathrm{N}$-acetylcystine $(2 \mathrm{mg} / \mathrm{ml})$. A non-traumatic steel tipped catheter (Olympus UK PW5V-1) was then used to apply small drops of $0.05 \%$ CV onto the surface of the lesion followed by a "fixing" phase of 1-2 minutes. High magnification views of the lesion were then acquired.

\section{Tissue sampling}

All identified lesions diagnosed using HMCC were biopsied, removed by endoscopic mucosal resection (EMR), or referred for surgical resection. The following criteria were used to guide the appropriate diagnostic or therapeutic technique and subsequent referral for surgical intervention.

\section{Biopsy only}

- Lesions with a dominant pit type I/II (unless exceeding $10 \mathrm{~mm}$ in diameter or showing atypical morphology such as a depressed area). Such lesions are often associated with hyperplastic/metaplastic histology only. ${ }^{12}$

- Lesions demonstrating asymmetrical lift or the non-lifting sign of Uno (associated with submucosal desmoplasis where EMR can be complicated by perforation and bleeding). ${ }^{29}$

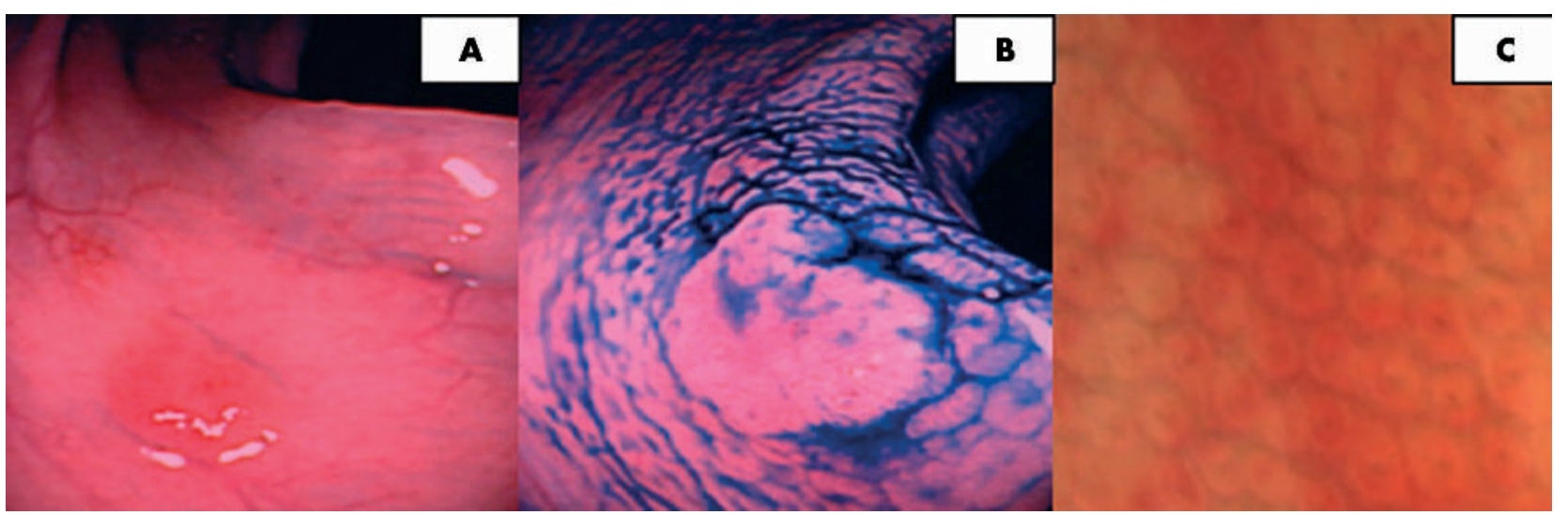

Figure 1 (A) Focal erythema noted at the mid transverse colon (conventional views). (B) Indigo carmine chromoscopy clearly delineates the circumferential margin of the lesion. The lesion is flat with slight elevation (JRSC Ila). No depressed component is apparent. (C) High magnification chromoscopic colonoscopy ( $100 \times$ magnification) shows a type I pit pattern. The lesion was hyperplastic at histology. 


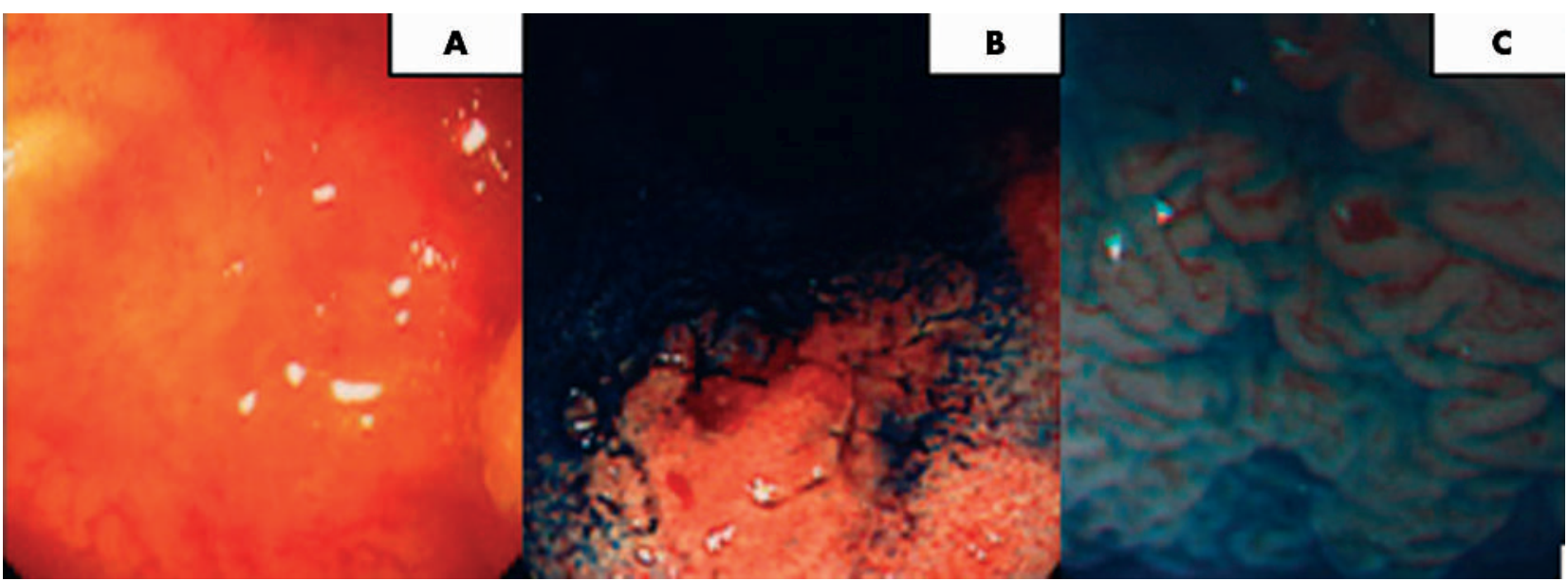

Figure 2 (A) Conventional views of the proximal ascending colon. The vascular net pattern is disrupted with central pallor and peripheral erythema. (B) Indigo carmine chromoscopy applied at the site of subtle mucosal abnormality in the distal ascending colon. A flat type llb lesion is now well demarcated (non-magnified views). (C) High magnification chromoscopic colonoscopy views (100× magnification). A type IIIL pit pattern is observed. The lesion was an F-type lateral spreading tumour or carpet lesion with low grade dysplasia adenomatous histology.

- Lesions spreading over two consecutive folds or occupying in excess of $33 \%$ of the luminal circumference.

- Lesions where anatomical position made endoscopic access for EMR impossible.

- Lesions showing an invasive pit pattern at CV chromoscopy (associated with neoplastic invasive disease). ${ }^{12}$

\section{Therapeutic EMR}

- Lesions with a dominant type IIIL, IIIs, or type IV pit.

- None of the above criteria fulfilled.

Lesions that did not fulfil criteria for EMR and which were considered clinically "high risk" had an adjacent submucosal tattoo of Indian ink applied to facilitate further localisation if required.

\section{Image capture}

All procedures were digitally recorded and time analysed (intubation/extubation/therapeutics) using a Macintosh G4 interface to an analogue-digital PCX 540 transducer. Exporting software was I-Movie version 2.1 for Mac OS10 that allowed the entire recording to be written to nonrewritable Sony CDQ74N2 $650 \mathrm{MB}$ disks in Quick Time streaming mode. All images could therefore be reviewed following the procedure if required.

\section{Histopathology}

A single consultant histopathologist blinded to the morphological description or pit pattern analysis identified by the colonoscopist examined all retrieved tissue. Submucosal and carcinoid tumours were excluded from analysis as the predominant submucosal localisation of such lesions does

Table 1 Modified Kudo criteria for the classification of colorectal pit patterns

\begin{tabular}{|c|c|c|c|}
\hline $\begin{array}{l}\text { Pit } \\
\text { type }\end{array}$ & Characteristics & $\begin{array}{l}\text { Appearance } \\
\text { using HMCC }\end{array}$ & $\begin{array}{l}\text { Pit size } \\
(\mathrm{mm})\end{array}$ \\
\hline I & Normal round pits & & $0.07(0.02)$ \\
\hline II & Stella or papillary & & $0.09(0.02)$ \\
\hline Ills & Tubular round pits, smaller than pit type I & & $0.03(0.01)$ \\
\hline IIIL & Tubular/large & & $0.22(0.09)$ \\
\hline IV & Sulcus/gyrus & & $0.93(0.32)$ \\
\hline$V(a)$ & Irregular arrangement and sizes of IIIL, IIIs, IV type pit & & N/A \\
\hline
\end{tabular}




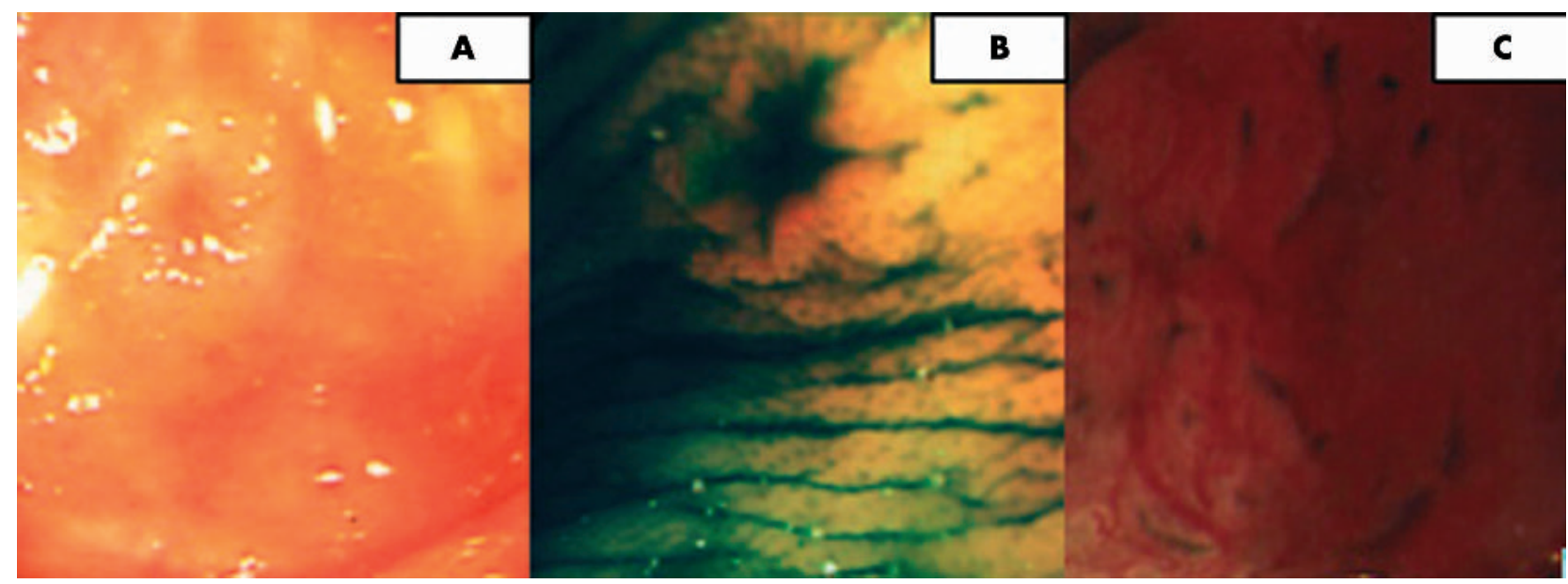

Figure 3 (A) JRSC type lla/c (flat elevation with central depression) seen using conventional views in the distal transverse colon. (B) Indigo carmine chromoscopy shows pooling of dye in the area of central depression. (C) Crystal violet chromoscopy at $100 \times$ magnification. A type IIIL pattern is seen at the periphery of the lesion with an absent amorphic pit pattern centrally.

not disrupt the mucosal colonic pit pattern. Specimens were immediately fixed in $10 \%$ buffered formalin solution and examined using haematoxylin and eosin staining. The Vienna criteria were used to define dysplasia as either low grade (LGD) or high grade (HGD). ${ }^{30}$ Within the HGD group was intramucosal carcinoma and carcinoma in situ. Neoplastic extension vertically into the submucosal layer 3 or beyond was classed as invasive advanced cancer (T2 disease). ${ }^{30}$

\section{Statistical analysis}

Statistical differences were analysed by $\chi^{2}$ tests of independence, the Mann-Whitney U test, and Fisher's exact test. A $p$ value of less than 0.05 was considered statistically significant. All calculations were made using the SPSS statistics package for Macintosh (system OsX 2.1-Microsoft Corp., USA).

\section{RESULTS}

\section{Clinical data}

A total of 1850 patients underwent colonoscopy. Males represented $833(45 \%)$ of the cohort with a mean age of 63 years (range 16-92). Caecal intubation or insertion to the anastomosis in patients with a previous right hemicolectomy was possible in 1795 (97\%) patients, with 1739 (94\%) receiving confirmatory terminal-ileal intubation and biopsy. Mean diagnostic extubation time (excluding therapeutic procedures) was 24 minutes (range 14-54). All patients received a single $20 \mathrm{mg}$ intravenous bolus of buscopan (20 mg) unless contraindicated at initial anal intubation. Sedation using intravenous midazolam was required in 795 (43\%) cases (mean dose $2.5 \mathrm{mg}$ (range 0.5-10)). One sigmoid perforation occurred post-EMR and two significant bleeds required transfusion. No complications required operative intervention and there were no deaths.

\section{Demographical data: flat and depressed lesions}

A total of 1008 lesions were identified from 1624 (88\%) patients. A single lesion was found in 840 (52\%) cases with $314(19 \%)$ and $56(3 \%)$ patients having two or more than three lesions, respectively. A total of 381 lesions (38\%) were hyperplastic/metaplastic and $616(61 \%)$ adenomatous. Eleven adenocarcinomas were diagnosed (stage T2 or beyond), representing $1 \%$ of all lesions.

Three hundred and sixty two (95\%) flat hyperplastic lesions (mean size $7.5 \mathrm{~mm}$ (range 1-16)) were located within the left colon and none had a depressed component at chromoscopy. In comparison, of the 616 adenomas (mean size $8 \mathrm{~mm}$ (range 4-38)) and invasive carcinomas (mean size $21 \mathrm{~mm}$ (range 1-38)) detected, 455 (74\%) and 9 (82\%), respectively, were located in the right colon. All of the invasive carcinomas ( $\mathrm{T} 2$ or beyond) had an area of depression noted at chromoscopy.

\section{Association of HMCC pit pattern analysis and histology}

Table 2 shows the association of pit patterns obtained using HMCC compared with actual histopathological diagnosis. The percentages of neoplastic change in lesions demonstrating a Kudo type I, II, IIIL, IIIs, IV, and V(a/n) were $2 \%$, 5\%, 93\%, $82 \%, 99.5 \%$, and $98 \%$, respectively. A total of $11(3 \%)$ and $3(1 \%)$ adenomas with LGD and HGD were classed as nonneoplastic at HMCC, showing a type I/II pit pattern. Villous adenomas with focal carcinoma limited to the submucosal layer 1 were found in $6(3 \%)$ lesions with a type IV pit pattern. Eleven $(100 \%)$ of the invasive carcinomas (T2 or beyond) showed an invasive pit pattern (IIIs/V) at HMCC.

\section{Clinical applicability and receiver operator characteristics}

Table 3 shows the association between pit patterns and histopathology when subgrouped into neoplastic (IIIL, IV, IIIs, $\mathrm{V}(\mathrm{a} / \mathrm{n})$ and non-neoplastic (I, II) classes. The sensitivity of HMCC and pit pattern analysis at distinguishing neoplastic from non-neoplastic lesions was $98 \%$, with a positive predictive value of $95 \%$. Specificity, negative predictive value, and overall accuracy were $92 \%, 96 \%$, and $95 \%$ respectively.

Table 4 shows a subgroup analysis of pit patterns according to the neoplastic/non-invasive (IIIL/IV) and neoplastic/ invasive (IIIs/V) types. When using HMCC to differentiate these two groups, the calculated sensitivity, specificity, positive predictive value, negative predictive value, and accuracy were $50 \%, 98 \%, 91 \%, 86 \%$, and $87 \%$ respectively.

The diagnostic accuracy of HMCC in predicting neoplastic from non-neoplastic disease was not significantly influenced by size or morphological classification according to $\chi^{2}$ testing. Although the diagnostic accuracy of lesions measuring more than $8 \mathrm{~mm}$ in diameter was slightly lower than for lesions $<8 \mathrm{~mm}$ in diameter, this did not reach statistical significance $(\mathrm{p}>0.5)$.

\section{DISCUSSION}

The first reported use of HMCC was in 1978 by Tada et al who demonstrated improved endoscopic definition of extent and 
Table 2 Association of pit patterns using high magnification chromoscopic colonoscopy compared with histopathology

\begin{tabular}{|c|c|c|c|c|c|c|c|}
\hline \multirow[b]{2}{*}{ Histology } & \multicolumn{7}{|l|}{ Pit pattern } \\
\hline & 1 & II & IIIL & IIIs & IV & $\mathrm{V}(\mathrm{a} / \mathrm{n})$ & Total \\
\hline Hyperplastic/metaplastic & 126 & 223 & 24 & 6 & 1 & 1 & $381(38 \%)$ \\
\hline Adenoma (LGD) & 2 & 9 & 296 & 5 & 169 & 2 & $483(48 \%)$ \\
\hline Adenoma (HGD) & 0 & 3 & 11 & 21 & 60 & 38 & $133(13 \%)$ \\
\hline Carcinoma (T2 or beyond) & 0 & 0 & 0 & 1 & 0 & 10 & $11(1 \%)$ \\
\hline Total & $128(13 \%)$ & $235(23 \%)$ & $331(33 \%)$ & $33(3 \%)$ & $230(23 \%)$ & $51(5 \%)$ & $1008(100 \%$ \\
\hline
\end{tabular}

HGD, high grade dysplasia; LGD, low grade dysplasia.

severity in inflammatory bowel disease. ${ }^{31}$ Subsequently, Nishizawa showed that discrete and characteristic pit patterns were present in minute colorectal carcinomas, flat adenomas, and mucosal carcinoma using HMCC. ${ }^{32}$ Kato et al's retrospective review of 4445 patients undergoing HMCC examined 3438 lesions (classified according to the modified Kudo criteria) and compared them with either endoscopic or surgically resected specimens. ${ }^{13}$ The diagnostic accuracies in this series for non-neoplastic lesions, adenomas, and invasive carcinomas were $75 \%, 94 \%$, and $85 \%$, respectively. ${ }^{13}$ Despite the large number of lesions examined, histological translation to Western practice in this series was complicated by an adapted Vienna criteria classification, which in the cancer group included only cases with submucosal invasion and the large group designated adenomatous failed to differentiate the grade of dysplasia. ${ }^{13}$ This fact has major clinical implications when adopting follow up surveillance strategies where the malignant potential of LGD is low. ${ }^{33}$ This study confirmed previous reporting of morphology and pit pattern characteristics demonstrated by flat and depressed colorectal lesions. $^{3435}$

Konishi et al reported the first randomised trial comparing magnifying and non-magnifying chromoscopic colonoscopy to discriminate between neoplastic and non-neoplastic colorectal lesions. ${ }^{36}$ The accuracy of magnifying colonoscopy in distinguishing neoplastic from non-neoplastic lesions (92\%) was significantly higher than for non-magnifying colonoscopy $(68 \%) .{ }^{36}$ These data therefore showed magnifying colonoscopy to be superior to non-magnifying colonoscopy for distinguishing between non-neoplastic and non-neoplastic lesions, and hence provided the rationale for using HMCC in this study.
Two prospective studies have addressed the efficacy of HMCC at differentiating neoplastic from non-neoplastic colorectal lesions. ${ }^{21} 22$ Tung et al assessed 175 polyps from 141 consecutive patients. ${ }^{21}$ Although not described in the methodology, differentiation of the neoplastic type pit patterns (IIIs/V) can be enhanced by the use of CV staining post-mucolysis. ${ }^{25}$ The use of $\mathrm{CV}$ in this study may have improved neoplastic sensitivity rates and aided diagnosis in the six neoplastic lesions that were misclassified. Our study used CV staining to aid diagnosis of the invasive crypt types which may have influenced the improved sensitivity (98\%) and specificity (92\%) when differentiating neoplastic from non-neoplastic lesions as compared with Tung ${ }^{21}(93.8 \%$ and $64.6 \%$, respectively). Furthermore, CV staining for diagnosis of the invasive pit patterns was used in Togashi et al's series ${ }^{22}$ of 923 lesions with a comparable sensitivity (92\%) but improved specificity $(73.3 \%)$ as compared with Tung's data. ${ }^{21}$

The lower overall specificity in both studies compared with our data may have also been influenced by endoscopic experience. Although not statistically significant, the specificity in Tung's analysis improved as the operator experience of pit patterns accumulated. ${ }^{21}$ A similar trend was noted in Togashi's series where the authors concluded that experience of 200 lesions assessed using HMCC was required to overcome the learning curve. ${ }^{22}$ The colonoscopist in our study had experience of over 800 lesions prior to commencing this study. Additionally, our data support the observation by Tung et al that diagnostic accuracy is not influenced by lesion size. ${ }^{21}$

No other prospective studies have addressed the ability of HMCC and pit pattern analysis to differentiate neoplastic non-invasive from neoplastic invasive lesions. In our series, despite a high specificity (98\%), sensitivity was poor (50\%).

Table 3 Association of pit pattern and histopathology when subgrouped into nonneoplastic and neoplastic subgroups

\begin{tabular}{lcccc}
\hline Pit pattern & $\begin{array}{l}\text { Hyperplastic/ } \\
\text { metaplastic }\end{array}$ & $\begin{array}{l}\text { Adenoma } \\
\text { (LDD) }\end{array}$ & $\begin{array}{l}\text { Adenoma } \\
\text { (HGD) or beyond }\end{array}$ & Total \\
\hline Non-neoplastic I/II & 349 & 11 & 3 & $363(36 \%)$ \\
Neoplastic IIIL/IIIs/IV/V & 32 & 472 & 141 & $645(64 \%)$ \\
Total & $381(38 \%)$ & $483(48 \%)$ & $144(14 \%)$ & $1008(100 \%)$ \\
\hline
\end{tabular}

HGD, high grade dysplasia; LGD, low grade dysplasia.

Table 4 Subgroup analysis of pit patterns according to neoplastic/non-invasive and neoplastic/invasive types

\begin{tabular}{|c|c|c|c|}
\hline \multirow[b]{2}{*}{ Pit pattern } & \multicolumn{3}{|l|}{ Histology } \\
\hline & $\begin{array}{l}\text { Adenoma } \\
\text { LGD }\end{array}$ & $\begin{array}{l}\text { Adenoma HGD or } \\
\text { beyond }\end{array}$ & Total \\
\hline $\begin{array}{l}\text { Neoplastic/non invasive IIIL/IV } \\
\text { Neoplastic/invasive IIIs/V } \\
\text { Total }\end{array}$ & $\begin{array}{l}465 \\
7 \\
472(77 \%)\end{array}$ & $\begin{array}{l}71 \\
70 \\
141(23 \%)\end{array}$ & $\begin{array}{l}536(87 \%) \\
77(13 \%) \\
613(100 \%)\end{array}$ \\
\hline
\end{tabular}

HGD, high grade dysplasia; LGD, low grade dysplasia. 
Therefore, when applied to this clinical scenario, the probability that a lesion had HGD or beyond as compared with adenoma (LGD) was poorly predicted using HMCC and is a limitation of this technique.

Finally, in addition to detection and diagnosis of sporadic neoplastic lesions of the colorectum, chromoscopic colonoscopy and pit pattern analysis have recently been described for the detection of intraepithelial neoplasia, and extent and severity assessment in chronic ulcerative colitis. ${ }^{37}$ In the randomised controlled trial of Kiesslich et al, significantly more intraepithelial neoplastic lesions were detected using methylene blue chromoscopy as compared with conventional colonoscopy and serial quadrantic biopsies (32 v 10; $\mathrm{p}=0.003) .{ }^{37}$ In the chromoscopy group there was also a significantly better correlation between the endoscopic assessment of degree $(p=0.0002)$ and extent $(89 \%$ v $52 \%$; $\mathrm{p}<0.0001$ ) of colonic inflammation and histopathological findings as compared with conventional colonoscopy. ${ }^{37}$ Furthermore, using the modified pit pattern classification, both the sensitivity and specificity for differentiating between non-neoplastic and neoplastic lesions was $93 \%{ }^{37}$ These data further suggest that chromoscopic colonoscopy is a novel tool for the early detection of intraepithelial neoplasia and CRC in patients undergoing screening colonoscopy with ulcerative colitis.

\section{CLINICAL RECOMMENDATIONS}

HMCC and pit pattern analysis is a useful tool when discriminating between neoplastic and non-neoplastic flat and depressed colorectal lesions. The decision to progress to therapeutic intervention using EMR can be guided using HMCC, avoiding inappropriate attempted endoscopic resection of lesions without a malignant potential or those which should be referred for surgical excision. We therefore recommend the following endoscopic strategies based on HMCC and pit pattern analysis.

- Small $(<10 \mathrm{~mm}$ ) flat lesions without a depressed component and showing a type I/II pit pattern can be left in situ without biopsy.

- Flat lesions (non-depressed) with a type IIIL/IV pit pattern can be resected in a single step procedure. This combines histological diagnosis and treatment. A submucosal tattoo should be placed adjacent to the resection site to permit future localisation. Inclusion in a systematic surveillance programme of 3-5 year intervals should then commence.

- For lesions with a depressed component and a type IIIs/V pit pattern, either alone or in combination, should receive cold biopsy only, even if small, and adjacent mucosa tattooed for future localisation. Further evaluation of such lesions using a $20 \mathrm{MHz}$ ultrasound mini probe (USMP) may be helpful in assessing the invasive depth of the lesion and also improve safety if elective EMR is performed (tethering to the underlying muscularis mucosa can be clearly seen using USMP, where the risk of perforation or incomplete resection is marked). ${ }^{38} 39$ Further studies however are required.

In conclusion, this is the largest prospective study addressing the efficacy of HMCC and pit pattern analysis in differentiating neoplastic from non-neoplastic lesions of the colorectum. We have shown that the technique has a high overall accuracy in this clinical context. However, the technique is not $100 \%$ sensitive or specific, and although a useful diagnostic tool in vivo, is not a complete replacement for histopathology. It is important for this technology to be used and developed but also requirements for further education and colonoscopic training need to be addressed. ${ }^{40}$ In experienced hands however HMCC represents a significant advance in colonoscopic practice, which may improve diagnostic yield of significant lesions, lower the burden of insignificant biopsies interpreted by pathologists, and enhance therapeutic safety. All of the above are essential requisites with the introduction of a colorectal cancer screening programme in the UK where economic resources and manpower are scarce.

\section{ACKNOWLEDGEMENTS}

This work was funded by the following charitable organisations: the Smith and Nephew Endoscopy Research Fellowship; BRET Research Foundation; Mason Medical Foundation; and Special Trustees to the Royal Hallamashire Hospital Sheffield.

\section{Authors' affiliations}

D P Hurlstone, D S Sanders, A J Lobo, Gastroenterology and Liver Unit, Royal Hallamshire Hospital, Sheffield, UK

S S Cross, Academic Unit of Pathology, Section of Oncology and Pathology, Division of Genomic Medicine, University of Sheffield Medical School, Sheffield, UK

I Adam, A J Shorthouse, S Brown, Academic Department of Surgery, Royal Hallamshire Hospital, Sheffield, UK

\section{REFERENCES}

1 Morson BC. President's address. The polyp-cancer sequence in the large bowel. Proc R Soc Med 1974:67:451-7.

2 Morson BC. Evolution of cancer of the colon and rectum. Cancer 1974;34(suppl):845-9.

3 Winawer SJ, Zauber AG, Ho MN, et al. Prevention of colorectal cancer by colonoscopic polypectomy. The National Polyp Study Workgroup. NEngl J Med 1993;329:1977-81.

4 Kudo S, Kashida H, Tamura T, et al. Colonoscopic diagnosis and management of nonpolypoid early colorectal cancer. World J Surg 2000;24:1081-90.

5 Tada S, lida M, Matsumoto T, et al. Small flat cancer of the rectum: clinicopathological and endoscopic features. Gastrointest Endosc 1995:42:109-13.

6 Hurlstone DP, Cross SS, Adam I, et al. A prospective clinicopathological and endoscopic evaluation of flat and depressed colorectal lesions in the UK. Am J Gastroenterol 2003;98:2543-9.

7 Rembacken BJ, Fujii T, Cairns A, et al. Flat and depressed colonic neoplasms: a prospective study of 1000 colonoscopies in the UK. Lancet 2000;355:1211-14.

8 Saitoh Y, Waxman I, West AB, et al. Prevalence and distinctive biologic features of flat colorectal adenomas in a North American population. Gastroenterology 2001;120:1657-65.

9 Tsuda S, Veress B, Toth E, et al. Flat and depressed colorectal tumours in a southern Swedish population: a prospective chromoendoscopic and histopathological study. Gut 2002;51:550-5.

10 Adachi M, Okinaga K, Muto T. Flat adenoma of the large bowel: reevaluation with special reference to central depression. Dis Colon Rectum 2000;43:782-7.

11 Wolber RA, Owen DA. Flat adenoma of the colon. Hum Pathol 1991;22:70-4.

12 Kudo S, Rubio CA, Teixeira CR, et al. Pit pattern in colorectal neoplasia: endoscopic magnifying view. Endoscopy 2001;33:367-73.

13 Kato S, Fujii T, Koba l, et al. Assessment of colorectal lesions using magnifying colonoscopy and mucosal dye spraying: can significant lesions be distinguished? Endoscopy 2001;33:306-10.

14 Haggitt RC, Glotzbach RE, Soffer EE, et al. Prognostic factors in colorectal carcinomas arising in adenomas: implications for lesions removed by endoscopic polypectomy. Gastroenterology 1985;89:328-36.

15 Konishi F, Morson BC. Pathology of colorectal adenoma: a colonoscopic survey. J Clin Pathol 1982;35:830-41.

16 Hamilton SR. The adenoma-adenocarcinoma sequence in the large bowel: variations on a theme. J Cell Biochem Suppl 1992;16:41-6.

17 Kudo S, Tamura S, Hirota S, et al. The problem of de novo colorectal carcinoma. Eur J Cancer 1995;31:1118-20.

18 Kurahashi T, Kaneko K, Makino R, et al. Colorectal carcinoma with special reference to growth pattern classifications: clinicopathological characteristics and genetic changes. J Gastroenterol 2002;37:354-62.

19 Kodaira S, Yao T, Nakamura K. The incidence of lymph node metastasis of submucosal colorectal carcinomas in each submucosal invasion depth degree. Results from a questionnaire survey (in Japanese with English abstract). Stomach Intest 1994;29:1 137-42.

20 Bedenne L, Faivre J, Boutron MC, et al. Adenoma-carcinoma sequence or "de novo" carcinogenesis? A study of adenomatous remnants in a populationbased series of large bowel cancers. Cancer 1992;69:883-8.

21 Tung SY, Wu CS, Su MY, et al. Magnifying colonoscopy in differentiating neoplastic from nonneoplastic colorectal lesions. Am J Gastroenterol $2001 ; 96: 2628-32$ 
22 Togashi K, Konishi F, Ishizuka T, et al. Efficacy of magnifying endoscopy in the differential diagnosis of neoplastic and non-neoplastic polyps of the large bowel. Dis Colon Rectum 1999;42:1602-8.

23 Hurlstone DP. High-resolution magnification chromoendoscopy: common problems encountered in "pit pattern" interpretation and correct classification of flat colorectal lesions. Am J Gastroenterol 2002;97:1069-70.

24 Riddell RH, Iwafuchi M. Problems arising from eastern and western classification systems for gastrointestinal dysplasia and carcinoma: are they resolvable? Histopathology 1998;33:197-202.

25 Hurlstone DP, Fujii T, Lobo AJ. Early detection of colorectal cancer using high magnification chromoscopic colonoscopy. Br J Surg 2002;89:272-82.

26 Japanese Research Society for Cancer of the Colon and Rectum. General rules for clinical and pathological studies on cancer of the colon, rectum and anus. Part I. Clinical classification. Jpn J Surg 1983;13:557-73.

27 Japanese Research Society for Cancer of the Colon and Rectum. General rules for clinical and pathological studies on cancer of the colon, rectum and anus. Part II. Histopathological classification. Jpn J Surg 1983;13:574-98.

28 Sawada T, Hojo K, Moriya Y. Colonoscopic management of focal and early colorectal carcinoma. Baillieres Clin Gastroenterol 1989;3:627-45.

29 Uno Y, Munakata A. The non-lifting sign of invasive colon cancer. Gastrointest Endosc 1994;40:485-9

30 Schlemper RJ, Riddell RH, Kato Y, et al. The Vienna classification of gastrointestinal neoplasia. Gut 2000;47:251-5.

31 Tada M, Misaki F, Kawai K. A new approach to the observation of minute changes of the colonic mucosa by means of magnifying colonoscope, type CFMB-M (Olympus). Gastrointest Endosc 1978;24:146-7.
32 Nishizawa M. Pertaining to histopathogenesis, growth and progression of early cancer of the colon and rectum in terms of dissecting microscopy and clinical aspects. Stomach Intestine 1985;20:1036-41.

33 Atkin WS, Morson BC, Cuzick J. Long-term risk of colorectal cancer after excision of rectosigmoid adenomas. N Engl J Med 1992;326:658-62.

34 Jaramillo $E$, Watanabe $M$, Slezak P, et al. Flat neoplastic lesions of the colon and rectum detected by high-resolution video endoscopy and chromoscopy. Gastrointest Endosc 1995;42:114-22.

35 Kudo S, Hayashi S, Miura K, et al. The clinicopathological features of flat and depressed type of early colorectal cancer (in Japanese with English abstract). Stomach Intestine 1989;24:317-29.

36 Konishi K, Kaneko K, Kurahashi T, et al. A comparison of magnifying and nonmagnifying colonoscopy for diagnosis of colorectal polyps: A prospective study. Gastrointest Endosc 2003:57:48-53.

37 Kiesslich R, Fritsch J, Holtmann M, et al. Methylene blue-aided chromoendoscopy for the detection of intraepitheial neoplasia and colon cancer in ulcerative colitis. Gastroenterology 2003;124:880-8.

38 Waxman I, Saitoh Y, Raju GS, et al. High-frequency probe EUS-assisted endoscopic mucosal resection: a therapeutic strategy for submucosal tumors of the GI tract. Gastrointest Endosc 2002;55:44-9.

39 Saitoh Y, Obara T, Einami K, et al. Efficacy of high-frequency ultrasound probes for the preoperative staging of invasion depth in flat and depressed colorectal tumors. Gastrointest Endosc 1996:44:34-9.

40 Hurlstone DP, Brown S. Evidence to support high-magnification chromoscopic colonoscopy: evaluation of the Kudo type $\mathrm{V}$ crypt pattern with reference to flat and depressed colorectal lesions and screening implications for the UK. Gastrointest Endosc 2003;58:164.

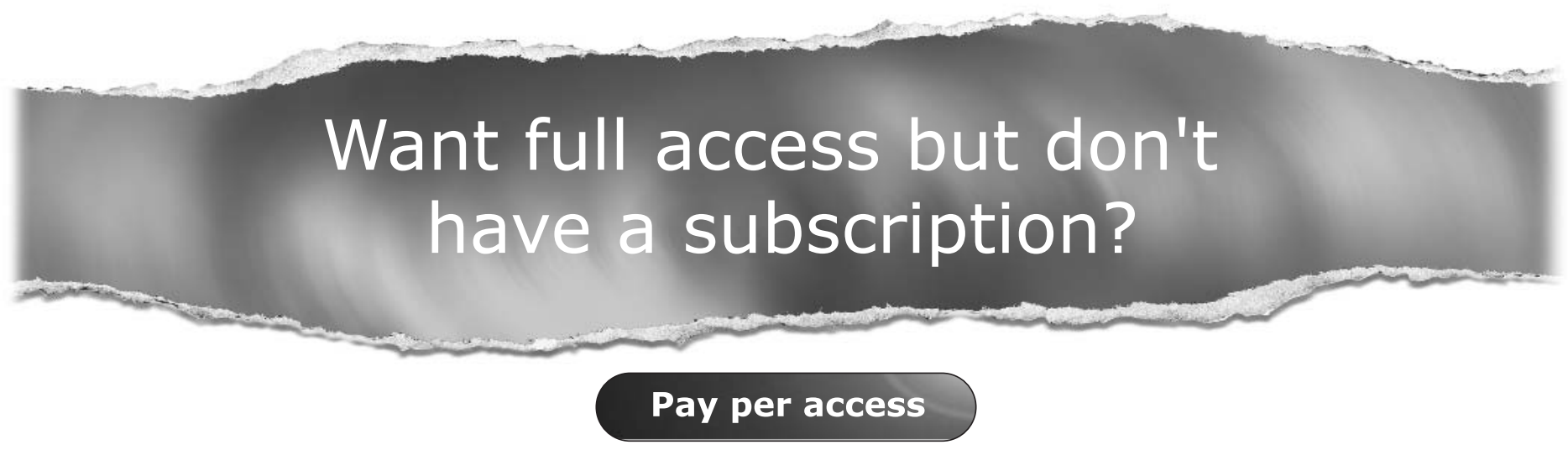

For just US $\$ 25$ you can have instant access to the whole website for 30 days. During this time you will be able to access the full text for all issues (including supplements) available. You will also be able to download and print any relevant pdf files for personal use, and take advantage of all the special features Gut online has to offer.

\section{www.gutjnl.com}

